\title{
A phase I dose-escalation study of PEP02 (irinotecan liposome injection) in combination with 5-fluorouracil and leucovorin in advanced solid tumors
}

Nai-Jung Chiang ${ }^{1,2}$, Tsu-Yi Chao ${ }^{3}$, Ruey-Kuen Hsieh ${ }^{4}$, Cheng-Hsu Wang ${ }^{5}$, Yi-Wen Wang ${ }^{6}$, C. Grace Yeh ${ }^{6}$ and Li-Tzong Chen ${ }^{1,2,7^{*}}$

\begin{abstract}
Background: PEP02 (also known as MM-398, nal-IRI) is a novel nanoparticle formulation of irinotecan encapsulated in liposomes. The aims of this study were to investigate the dose-limiting toxicity (DLT), maximum tolerated dose (MTD) and pharmacokinetics (PK) of PEP02 in combination with 5-FU and LV, in patients with advanced refractory solid tumors.
\end{abstract}

Methods: Patients were enrolled in cohorts to receive PEP02 from 60 to $120 \mathrm{mg} / \mathrm{m}^{2}$ (dose expressed as the irinotecan hydrochloride trihydrate salt) as a 90-min intravenous infusion on day 1, followed by $24 \mathrm{~h}$ infusion of 5 -FU $2,000 \mathrm{mg} / \mathrm{m}^{2}$ and LV $200 \mathrm{mg} / \mathrm{m}^{2}$ on days 1 and 8 , every 3 weeks.

Results: A total of 16 patients were assigned to four dose levels, 60 (three patients), 80 (six patients), 100 (five patients) and $120 \mathrm{mg} / \mathrm{m}^{2}$ (two patients). DLT was observed in four patients, two at the $100 \mathrm{mg} / \mathrm{m}^{2}$ dose level (one had grade III infection with hypotension and grade III hemorrhage; the other had grade III diarrhea and grade IV neutropenia), and two at the $120 \mathrm{mg} / \mathrm{m}^{2}$ dose level (one had grade III diarrhea and grade IV neutropenia; the other had grade III diarrhea). The MTD of PEP02 was determined as $80 \mathrm{mg} / \mathrm{m}^{2}$. The most common treatment-related adverse events were nausea (81\%), diarrhea (75\%) and vomiting (69\%). Among the six patients who received the MTD, one patient exhibited partial response, four patients had stable disease and one showed progressive disease. Pharmacokinetic data showed that PEP02 had a lower peak plasma concentration, longer half-life, and increased area under the plasma concentration-time curve from zero to time t of SN-38 than irinotecan at similar dose level.

Conclusions: The MTD of PEPO2 on day 1 in combination with 24-h infusion of 5-FU and LV on days 1 and 8 , every 3 weeks was $80 \mathrm{mg} / \mathrm{m}^{2}$, which will be the recommended dose for future studies.

Trial registration: The trial was retrospectively registered (NCT02884128) with date of registration: August 12, 2016.

Keywords: Liposomal irinotecan, 5-fluorouracil, Dose-limiting toxicity, Maximum tolerated dose

\footnotetext{
* Correspondence: leochen@nhri.og.tw; leochen@nhri.org.tw

${ }^{1}$ National Institute of Cancer Research, National Health Research Institutes, 2F,

No. 367, Sheng-Li Road, Tainan 704, Taiwan

${ }^{2}$ Division of Hematology/Oncology, Department of Internal Medicine,

National Cheng Kung University Hospital, Tainan, Taiwan

Full list of author information is available at the end of the article
} 


\section{Background}

PEP02 (also known as MM-398, nal-IRI) is an encapsulated nanoliposomal formulation of irinotecan hydrochloride (CPT-11) [1]. Irinotecan is a water-soluble semi-synthetic analogue of the natural alkaloid, camptothecin. It prevents DNA from unwinding and replication by inhibition of topoisomerase-I, and has already been approved for use worldwide. However, at higher dosage, irinotecan causes severe diarrhea and myelosuppression, which limits its therapeutic index. The therapeutic benefits of encapsulating anti-cancer drugs such as daunorubicin, doxorubicin and cytarabine in liposomes have been documented [2]. An appropriately designed liposome formulation may reduce the toxicity of cytotoxic agents to healthy tissues while maintaining its anti-tumor potency, which in turn improves treatment efficacy.

In our previous study, the maximum tolerated dose (MTD) of PEP02 monotherapy was found to be $120 \mathrm{mg} / \mathrm{m}^{2}$ at 3-week interval with favorable pharmacokinetic (PK) parameters of the active metabolite, SN-38 [3]. The acceptable toxicity profile explains the beneficial effects of PEP02 in combination with other cytotoxic agents. Irinotecan in combination with 5fluorouracil (5-FU) and leucovorin (LV) is the first-line or second-line therapy for locally advanced and metastatic colorectal cancer [4]. A synergistic effect was observed upon the sequential administration of irinotecan and 5FU $[5,6]$. On the basis of these results, the combination of PEP02 with 5-FU and LV is considered a reasonable approach to enhance their therapeutic efficacy. This Phase I dose escalation study aimed to investigate the MTD, doselimiting toxicity (DLT) and recommended dose of PEP02 in combination with 5-FU and LV.

Irinotecan is converted by carboxylesterases to its potent metabolite, $\mathrm{SN}-38$, which is detoxified in part by converting to inactive $\mathrm{SN}-38$ glucuronide (SN-38G) through UDP-glucuronosyl transferase 1A isoforms (UGT1A) [7]. The activity of UGT1A is related to gene polymorphism of UGT1A family members. Individuals with genetic mutations of UGT1A exhibit reduced glucuronidation of $\mathrm{SN}-38$ and an elevated risk of neutropenia and diarrhea compared with patients with wild-type alleles [8]. The correlation of UGT1A polymorphisms and toxicities is discussed.

\section{Methods}

\section{Patient eligibility}

This trial was a multi-center, open-label, Phase I, dose escalation study of PEP02 (PharmaEngine, Inc., Taipei, Taiwan) in combination with 5-FU and LV in patients with advanced solid tumors. The inclusion criteria were as follows: (1) histologically or cytologically confirmed advanced solid tumor refractory to standard systemic chemotherapy; (2) aged between 20 and 70 years; (3) Eastern Cooperative Oncology Group performance score (ECOG PS) of 0 or 1 ; (4) life expectancy $\geq 2$ months; (5) adequate bone marrow, hepatic and renal functions: white blood cells $\geq 3,000 / \mathrm{mm}^{3}$, absolute neutrophil count $\geq$ $1,500 / \mathrm{mm}^{3}, \quad$ platelets $\geq 100,000 / \mathrm{mm}^{3}, \quad$ hemoglobin $\geq$ $10 \mathrm{~g} / \mathrm{dL}$, serum total bilirubin within normal range, AST and ALT $\leq 3 \times$ upper limit of normal range, serum creatinine $\leq 1.5 \mathrm{mg} / \mathrm{dL}$ and blood urea nitrogen $\leq 25 \mathrm{mg} / \mathrm{dL}$; (6) no prior treatment for at least 4 weeks before study initiation, including major surgery, chemotherapy, any investigational products or radiotherapy (6 weeks for nitrosoureas and mitomycin C); (7) recovered from all treatment-related toxicities or resolved to no greater than grade 1 before enrollment; and (8) written informed consent.

The exclusion criteria were as follows: (1) known or suspicious primary or secondary brain tumors; (2) HBsAg-positive or anti-HCV antibody-positive with splenomegaly (defined as spleen size $>11 \mathrm{~cm}$ measured in longest diameter by CT scan); (3) uncontrolled active infection or other concomitant serious disease; (4) pregnancy or breast-feeding; (5) previous exposure to irinotecan; (6) history of allergic reactions to compounds of similar chemical or biologic composition as PEP02, 5FU, or LV. This trial was approved by the independent ethics committee of each participating institute and the Department of Health, Executive Yuan, Taiwan, and was performed in accordance with International Conference on Harmonization Good Clinical Practice guidelines and Good Clinical Laboratory Practice.

\section{Treatment and dose escalation schedule}

The study had a traditional $3+3$ design with threepatient cohorts for each dose level. Dose escalation was only performed after the successful completion of at least 1 full 3 -week cycle by each patient in the dosing cohort. If none of the first three patients experienced DLT, dose escalation was carried out for the next cohort of patients. If one of three patients developed DLT, the cohort was expanded to six patients. If two or more patients experienced any DLT, no more patients were to be entered at the current dose level and the lower dose level was to be declared the MTD. The MTD was the highest dose level with no more than 1 DLT among the accruals. A minimum of six patients were required to be tested at the dose level defined as the MTD. The starting dose of PEP02 was $60 \mathrm{mg} / \mathrm{m}^{2}$ with dose expressing as the irinotecan hydrochloride trihydrate salt, which was escalated by increments of $20 \mathrm{mg} / \mathrm{m}^{2}$ between dose levels. Each patient was assigned to a dose level, and no intra-patient dose escalation was allowed. 5-FU and LV were administered at a fixed dose of 2000 and $200 \mathrm{mg} / \mathrm{m}^{2}$, respectively. PEP02 was administered by intravenous 
infusion over 90 min on Day 1, followed by 24-h intravenous infusion of 5-FU and LV on days 1 and 8 every 3 weeks. Pre-medication included dexamethasone and a serotonin-antagonist. Prophylactic anti-cholinergic agent was not administered unless acute cholinergic reaction was observed in prior cycles of treatment. Anti-diarrhea agents were started according to the guideline of American Society of Clinical Oncology. Treatment was continued to a maximum of 6 cycles or until disease progression, unacceptable toxicity, treatment delay $>$ 2 weeks, or patient's refusal or death.

Dose modification on day 1 of subsequent cycles was only applied to PEP02, while the dosage of 5-FU/LV remained unchanged. All dose modifications were to be based on the worst proceeding toxicity. For patients who experienced $\geq$ grade 3 hematologic or non-hematologic toxicities, the dose of PEP02 was reduced by one dose level. In addition, the dose of 5-FU on day 8 of each cycle could be adjusted according to the laboratory data before the dosing. If the absolute neutrophil count (ANC) is between 1,000 and $1,499 / \mu \mathrm{L}$, platelet count is between 50,000 and $99,999 / \mu \mathrm{L}$, or diarrhea of grade 2 severity is observed, the dose of 5 -FU could be decreased by $25 \%$. 5-FU was withheld when $\mathrm{ANC}<999 / \mu \mathrm{L}$, platelet count $<50,000 / \mu \mathrm{L}$ or grade 3 diarrhea was observed. The conditions for the administration of the next cycle of treatment were $\mathrm{ANC} \geq 1,500 / \mu \mathrm{L}$, platelet counts $\geq$ $100,000 / \mu \mathrm{L}$, serum creatinine $\leq 1.5 \mathrm{mg} / \mathrm{dL}$, and full resolution of gastrointestinal toxicities.

\section{Definition of dose-limiting toxicity (DLT)}

Toxicities were assessed according to the National Cancer Institute's CTCAE version 3.0 (CTCAE, v3). DLT was defined as occurrence of 1 or more of the following events attributable to the study drugs during the first cycle: (1) grade III or IV non-hematological toxicity, except grade III nausea, vomiting, or anorexia; (2) grade IV hematologic toxicity lasting for $\geq 3$ days; (3) grade III hematologic toxicity associated with complications (e.g. neutropenic fever or bleeding); (4) dose delay of more than 2 weeks owing to drug-related toxicity. In addition, hematological assessment was performed daily whenever grade IV hematological toxicity occurred.

\section{Patient evaluation}

Pretreatment evaluations included medical history, physical examination, performance status, complete blood count, hepatic and renal functions and serology of HBsAg and anti-HCV antibody. Patients were evaluated weekly with complete blood count and biochemistry analysis. Radiologic studies to assess response were performed at baseline and then every 2 cycles of therapy according to the guidelines of Responses Evaluation Criteria in Solid Tumors criteria version 1.0. All complete and partial responses required confirmation by two consecutive observations at least 4 weeks apart.

\section{Pharmacokinetic sampling and analyzing}

During the first cycle of treatment, blood samples were collected before treatment, during the infusion at 30 and $60 \mathrm{~min}$, at the end of infusion, at1, 3, 9, 24, 48, 72 and $168 \mathrm{~h}$ after the end of infusion, and before the second cycle. Plasma levels of irinotecan and SN-38 were measured by validated LC/MS/MS analytical methods. The peak plasma concentration $\left(C_{\max }\right)$, time at which $C_{\max }$ occurred $\left(\mathrm{T}_{\max }\right)$, elimination half-life $\left(\mathrm{t}_{1 / 2}\right)$, area under the plasma concentration-time curve from zero to time $t$ $\left(\mathrm{AUC}_{0 \rightarrow \mathrm{t}}\right), \mathrm{AUC}$ through infinite time $\left(\mathrm{AUC}_{0 \rightarrow \infty}\right)$, and clearance (CL) were calculated. Pharmacokinetic parameters of individual data set were analyzed by a noncompartmental model by using WinNonlin ${ }^{\mathrm{Tm}}$ (Centara, St. Louis, MO).

\section{Pharmacogenetic studies}

Additional $5 \mathrm{~mL}$ blood sample was collected into a PAXgene vacutainer tube and DNA was extracted using a DNA purification kit. Fragment analysis was used for the detection of short tandem repeat polymorphism. The TaqMan-Allelic discrimination method or direct sequencing was used for the detection of single nucleotide polymorphisms, including UGT1A1*28 and UGT1A1*6.

\section{Statistical analysis}

The statistical analysis was descriptive and any inferential statistics was exploratory in nature. Summary statistics were provided for all efficacy, pharmacokinetic, pharmacogenetic, safety and baseline/demographic variables. For categorical variables, frequency tables including percentages were presented. For continuous variables, descriptive statistics such as number of available observations, mean with standard deviation (STD), minimum, and maximum were tabulated.

\section{Results}

\section{Patient characteristics, dose escalation, DLT and MTD}

Between March 2006 and August 2008, a total of 16 patients (seven men and nine women) were enrolled. The demographics and baseline characteristics of all patients are summarized in Table 1 . The median age was 49 years (range: 30-67 years). The most common primary tumors were pancreatic, stomach, and breast carcinomas. Other tumor types included keratinizing squamous cell carcinoma, cervical cancer and nasopharyngeal carcinoma. A total of 66 cycles of treatment were initiated, with an average of 4.1 cycles per patient (range: 1-6 cycles). There were seven patients (43.8\%) completed all 6 cycles of treatment. 
Table 1 Patient characteristics

\begin{tabular}{ll}
\hline Characteristic & Patients, n (\%) \\
\hline Patients enrolled & 16 \\
Age (yrs) & 49 \\
Median & $30-67$ \\
Range & \\
Sex & $7(44)$ \\
Male & $9(56)$ \\
Female & \\
ECOG performance status & $3(19)$ \\
0 & $13(81)$ \\
1 & \\
Tumor type & $4(25)$ \\
Breast cancer & $5(31)$ \\
Pancreatic cancer & $4(25)$ \\
gastric cancer & $3(19)$ \\
Other & \\
Previous treatment & $14(88)$ \\
Surgery & $9(56)$ \\
Radiotherapy & $16(100)$ \\
Chemotherapy &
\end{tabular}

Abbreviation: ECOG Eastern Cooperative Oncology Group

The dose escalation schedule is outlined in Table 2 . These patients were assigned to four dose levels, with three, six, five and two patients in dose level I, II, III, and IV, respectively. At first, none of the first three patients experienced DLT at dose level I, II, and III; therefore, the dose level was further escalated to $120 \mathrm{mg} / \mathrm{m}^{2}$. Because both of the initial two patients at $120 \mathrm{mg} / \mathrm{m}^{2}$ level experienced DLT during the first cycle of treatment (one had grade III diarrhea and grade IV neutropenia; the other had grade III diarrhea), three additional patients were recruited at the prior dose level, $100 \mathrm{mg} / \mathrm{m}^{2}$. However, both of the two newly accrued patients at $100 \mathrm{mg} / \mathrm{m}^{2}$ level experienced DLTs (one had grade III infection with hypotension and grade III hemorrhage; the other had grade III diarrhea and grade IV neutropenia), resulting in 2 episodes of DLT among the five patients at this dose level. Therefore, the tested dose level was further de-escalated to $80 \mathrm{mg} / \mathrm{m}^{2}$. Since none of the patients experienced any DLT, $80 \mathrm{mg} / \mathrm{m}^{2}$ of PEP02 by

Table 2 Dose escalation scheme

\begin{tabular}{llll}
\hline Dose Level & PEP02 $\left(\mathrm{mg} / \mathrm{m}^{2}\right)$ & No. patients & No. patients with DLT \\
\hline I & 60 & 3 & 0 \\
II & 80 & $3+3$ & $0+0$ \\
III & 100 & $3+2$ & $0+2$ \\
IV & 120 & 2 & 2 \\
\hline
\end{tabular}

Abbreviation: DLT dose-limiting toxicity 90-min intravenous infusion was determined as the MTD in combination with weekly infusion of 5-FU/LV on days 1 and 8 of a 21-day cycle.

\section{Toxicity}

All 16 patients were assessed for toxicity. Table 3 summarizes the therapy-induced toxicity during treatment. There were three (18.4\%) patients had grade III or above adverse events (AEs), and 13 and $0.2 \%$ of AEs led to dosing delay/reduction and permanent discontinuation of treatment, respectively. No treatment-related death was reported in the study.

The most common treatment-related AEs included nausea $(81.3 \%$ in incidence), followed by diarrhea (75.0\%), vomiting (68.8\%), fatigue (43.8\%), mucositis (mucosa inflammation, 43.8\%), leucopenia (37.5\%), neutropenia (37.5\%), weight loss (37.5\%), anemia (31.3\%), and alopecia $(31.3 \%)$. Acute cholinergic reaction was rarely observed. Compared with the entire safety population, patients who received $80 \mathrm{mg} / \mathrm{m}^{2}$, the MTD dose of PEP02 experienced less treatment-related AEs (51.1\% versus $57.6 \%$ ), as well as grade III or above AEs (10.6\% versus $18.4 \%$ ).

\section{Pharmacokinetics and exploratory pharmacogenetic studies}

The PK of PEP02 is shown in Table 4, Fig. 1a and b. CPT-11 and SN-38 were characterized for PEP02 single dose PK at dose levels of $60,80,100$, and $120 \mathrm{mg} / \mathrm{m}^{2}$ by 90-min intravenous infusion. Changes in the plasma concentration of CPT-11 showed almost the same pattern at all levels. All concentration curves of plasma CPT-11 peaked quickly and reached the maximum around $1 \mathrm{~h}$ after the end of PEP02 infusion and gradually dropped in a mono-exponential pattern until the last sampling point, which was similar to that observed for PEP02 monotherapy in a previous study [3]. At the MTD of PEP02, the Cmax of SN-38 was lower (7.98 $\pm 4.39 \mathrm{ng} / \mathrm{ml}$ ) than that of the conventional formulation of irinotecan at $125 \mathrm{mg} / \mathrm{m}^{2}(26.3 \pm 11.9 \mathrm{ng} / \mathrm{ml})$, whereas the AUC of SN-38 was higher than that of irinotecan (AUC0 $\rightarrow \mathrm{t}: \quad 343.36 \pm 133.24 \mathrm{ng} / \mathrm{ml}$ "h $\mathrm{vs}$. $229 \pm 108 \mathrm{ng} / \mathrm{mL} * \mathrm{~h})$. The $\mathrm{t}_{1 / 2}$ of SN-38 at the MTD of PEP02 was $57.54 \pm 17.81 \mathrm{~h}$, which was relatively longer than that of the conventional formulation $(10.4 \pm 3.1$ h). No statistically significant difference was observed in the mean values of all pharmacokinetic parameters of SN-38 among the 4 dose levels.

The majority of subjects showed wild type alleles for UGT1A1*28 (TA6TA6: 88\%) and UGT1A1*6 (GG: 69\%). No subject harbored homozygous mutation in UGT1A1*28 or UGT1A1*6 allele. Two and five patients had heterozygous UGT1A1*28 and UGT1A1*6, respectively. Of which, one patient with heterozygous 
Table 3 Treatment-emergent AEs with maximum CTC grade by dose level (incidence $\geq 20 \%$ )

\begin{tabular}{|c|c|c|c|c|c|}
\hline & Total $(N=16)$ & $\begin{array}{l}60 \mathrm{mg} / \mathrm{m}^{2} \\
N=3\end{array}$ & $\begin{array}{l}80 \mathrm{mg} / \mathrm{m}^{2} \\
N=6\end{array}$ & $\begin{array}{l}100 \mathrm{mg} / \mathrm{m}^{2} \\
N=5\end{array}$ & $\begin{array}{l}120 \mathrm{mg} / \mathrm{m}^{2} \\
N=2\end{array}$ \\
\hline $\mathrm{AE}$ & All grade & Grade 3-4 & & & \\
\hline Anemia & 7 (43.8\%) & 0 & 0 & $2(40 \%)$ & 0 \\
\hline Leukopenia & $6(37.5 \%)$ & 0 & 0 & $2(40 \%)$ & $1(50 \%)$ \\
\hline Neutropenia & $6(37.5 \%)$ & $1(33.3 \%)$ & $1(16.7 \%)$ & $2(40 \%)$ & $1(50 \%)$ \\
\hline Abdominal pain & 7 (43.8\%) & 0 & 0 & $1(20 \%)$ & $1(50 \%)$ \\
\hline Diarrhea & $12(75.0 \%)$ & 0 & $1(16.7 \%)$ & $2(40 \%)$ & $2(100 \%)$ \\
\hline Nausea & $13(81.3 \%)$ & 0 & $1(16.7 \%)$ & 0 & 0 \\
\hline Vomiting & $12(75.0 \%)$ & 0 & $1(16.7 \%)$ & 0 & 0 \\
\hline Fatigue & $8(50.0 \%)$ & 0 & 0 & $1(20 \%)$ & 0 \\
\hline Infection & $6(37.5 \%)$ & 0 & 0 & $2(40 \%)$ & $1(50 \%)$ \\
\hline Anorexia & $4(25.0 \%)$ & 0 & 0 & $1(20 \%)$ & 0 \\
\hline Hypoalbuminemia & $4(25.0 \%)$ & 0 & $1(16.7 \%)$ & 0 & 0 \\
\hline Hypokalemia & $8(50.0 \%)$ & 1 (33.3\%) & $2(33.3 \%)$ & $2(40 \%)$ & $1(50 \%)$ \\
\hline Hyponatremia & $4(25.0 \%)$ & 0 & 0 & $1(20 \%)$ & $1(50 \%)$ \\
\hline Cough & $5(31.3 \%)$ & $1(33.3 \%)$ & 0 & 0 & 0 \\
\hline
\end{tabular}

Abbreviation: $A E$ adverse event

UGT1A1*28 and UGT1A1*6 experienced grade IV neutropenia and grade III diarrhea, and had the largest dose-normalized AUC of SN-38. Four out of the 5 subjects with heterozygous $U G T 1 A 1 * 6$ possessed relatively higher dose-normalized AUC of SN-38 comparing to other subjects; of which 3 patients experienced grade III toxicities.

\section{Antitumor activity}

One patient at dose level III, who suffered from DLT did not complete at least one post-treatment tumor assessment. Among the 15 efficacy evaluable patients, two (13.3\%) had confirmed partial response (PR) and nine (60\%) had stable disease (SD), leading to the overall disease control rate (DCR) of $73.3 \%$. At the MTD of $80 \mathrm{mg} / \mathrm{m}^{2}, 1 \mathrm{PR}$ and 4 SD were observed among six patients. The tumor response rate and the disease control rate were 16.7 and $83.3 \%$, respectively. PR was observed in one gastric cancer patient (at the $80 \mathrm{mg} / \mathrm{m}^{2}$ dose level) and one breast cancer patient (at the $100 \mathrm{mg} / \mathrm{m}^{2}$ dose level).

\section{Discussion}

The current study evaluated the safety profile and preliminary efficacy of PEP02 in combination with 5-FU

Table 4 Pharmacokinetic parameters of PEP02 at each dose level

\begin{tabular}{|c|c|c|c|c|c|c|c|c|}
\hline & $\begin{array}{l}\text { Dose of PEP02 } \\
\left(\mathrm{mg} / \mathrm{m}^{2}\right)\end{array}$ & $\begin{array}{l}C_{\max } \\
\text { CPT-11 } \\
(\mu \mathrm{g} / \mathrm{mL}) \\
\mathrm{SN}-38 \\
(\mathrm{ng} / \mathrm{mL})\end{array}$ & $\begin{array}{l}T_{\max } \\
(\mathrm{hr})\end{array}$ & $\begin{array}{l}\mathrm{AUC}_{0 \rightarrow 169.5} \\
\mathrm{CPT}-11 \\
(\mathrm{hr}-\mu \mathrm{g} / \mathrm{mL}) \\
\mathrm{SN}-38 \\
(\mathrm{hr}-\mathrm{ng} / \mathrm{mL})\end{array}$ & $\begin{array}{l}\text { AUC }_{0 \rightarrow \infty} \\
\text { CPT-11 } \\
(\mathrm{hr}-\mu \mathrm{g} / \mathrm{mL}) \\
\mathrm{SN}-38 \\
(\mathrm{hr}-\mathrm{ng} / \mathrm{mL})\end{array}$ & $\begin{array}{l}V_{s s} \\
\left(\mathrm{~L} / \mathrm{m}^{2}\right)\end{array}$ & $\begin{array}{l}\mathrm{Cl} \\
\left(\mathrm{mL} / \mathrm{hr} / \mathrm{m}^{2}\right)\end{array}$ & $\begin{array}{l}t_{1 / 2} \\
(\mathrm{hr})\end{array}$ \\
\hline \multirow[t]{4}{*}{ Total CPT-11 } & $60, N=3$ & $28.9 \pm 15.8$ & $2.4 \pm 0.7$ & $1047 \pm 1210$ & $1047 \pm 1210$ & $2.80 \pm 1.59$ & $136 \pm 116$ & $21.1 \pm 11.7$ \\
\hline & $80, N=6$ & $29.2 \pm 5.2$ & $2.1 \pm 0.7$ & $1096 \pm 834$ & $1151 \pm 880$ & $3.39 \pm 0.74$ & $124 \pm 106$ & $33.3 \pm 15.1$ \\
\hline & $100, N=5$ & $44.1 \pm 7.7$ & $4.0 \pm 3.8$ & $2237 \pm 1090$ & $2289 \pm 1119$ & $2.86 \pm 0.75$ & $58 \pm 37$ & $43.17 \pm 4.8$ \\
\hline & $120, N=2$ & $47.9 \pm 16.2$ & $2.3 \pm 0.9$ & $1254 \pm 553$ & $1254 \pm 553$ & $3.95 \pm 0.83$ & $106 \pm 47$ & $54.4 \pm 17.4$ \\
\hline \multirow[t]{5}{*}{ SN-38 } & $60, N=3$ & $7.02 \pm 5.64$ & $13.1 \pm 11.7$ & $364 \pm 222$ & $1370 \pm 1122$ & NA & NA & $183.8 \pm 172.3$ \\
\hline & $80, N=6$ & $7.98 \pm 4.39$ & $13.3 \pm 18.3$ & $343 \pm 133$ & $505 \pm 165$ & NA & NA & $57.5 \pm 17.8$ \\
\hline & $100, N=5$ & $7.39 \pm 1.68$ & $12.2 \pm 12.3$ & $539 \pm 368$ & $840 \pm 433$ & NA & NA & $73.4 \pm 18.3$ \\
\hline & $120, N=2$ & $7.26 \pm 3.90$ & $37.8 \pm 17.2$ & $353 \pm 164$ & 305 & NA & NA & 30.8 \\
\hline & Irinotecan ${ }^{a}$ & $26.3 \pm 11.9$ & NA & $229 \pm 108$ & NA & NA & NA & $10.4 \pm 3.1$ \\
\hline
\end{tabular}

Mean \pm STD; $C_{\text {max }}$ peak concentration in plasma; $T_{\text {max }}$ time to achieve peak plasma concentration; $A U C_{0 \rightarrow 169.5}$ and $A U C_{0 \rightarrow \infty}$ area under the plasma concentration-time curve from time zero to $169.5 \mathrm{~h}$ and infinity, respectively; $\mathrm{V}_{s s}$, volume of distribution at steady state; $t_{1 / 2}$, plasma terminal elimination half-life; $\mathrm{Cl}_{1}$, total clearance of drug from plasma; NA, not available

arinotecan $125 \mathrm{mg} / \mathrm{m}^{2}$, package inset of Campto 

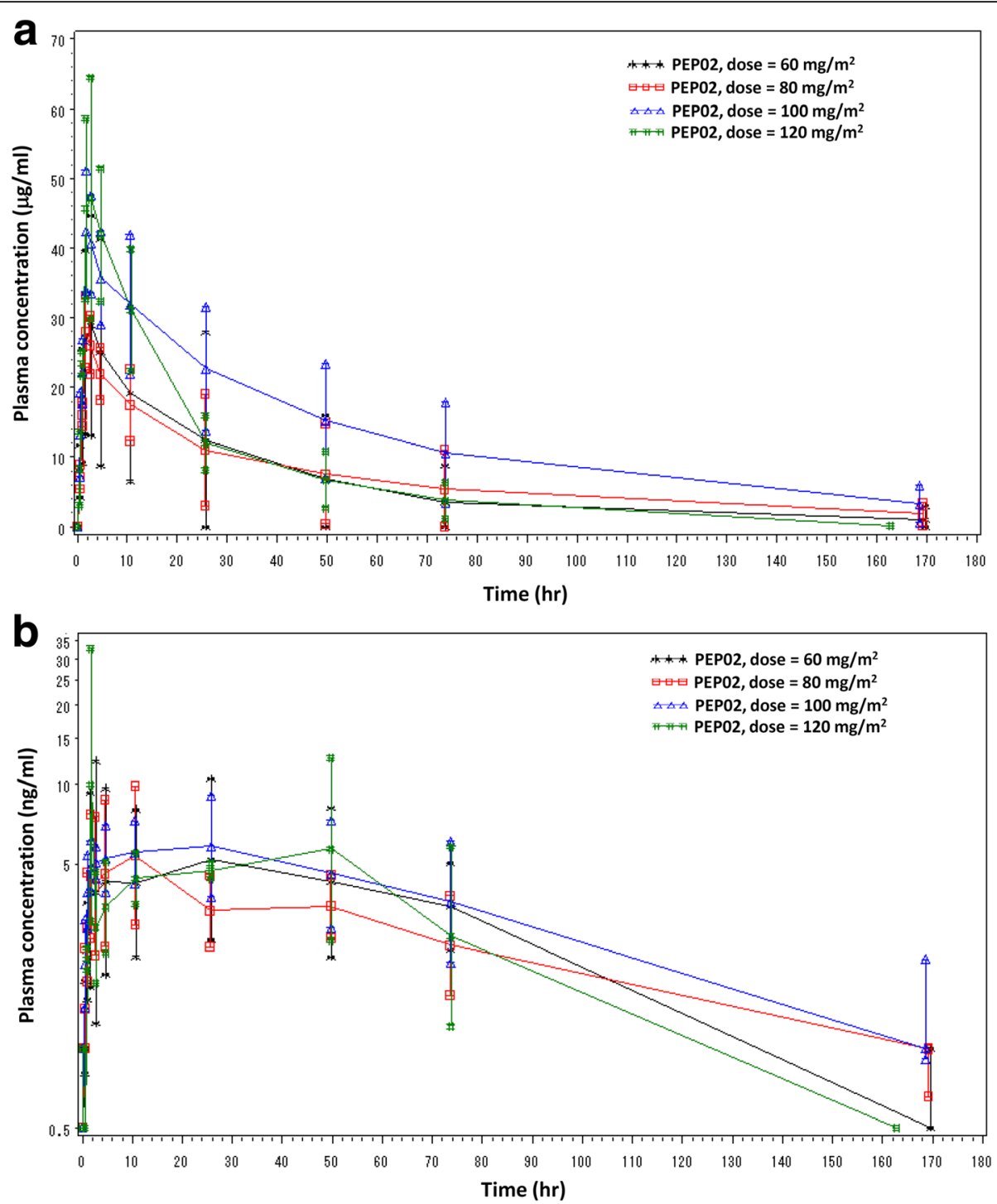

Fig. 1 Plasma concentration-time profiles of $\mathbf{a}$ CPT-11 and $\mathbf{b}$ SN-38 at different PEP02 doses

and LV, in patients with refractory advanced malignancy. Gastrointestinal toxicities and myelosuppression were the major DLTs, which were comparable to those of free irinotecan and PEP02 monotherapy $[3,9]$. The MTD $\left(80 \mathrm{mg} / \mathrm{m}^{2}\right)$ of PEP02, in combination with infusion of 5-FU and LV on days 1 and 8 of every-3-week schedule is recommended for the future studies. In a previous study, the MTD of PEP02 monotherapy with a 3-week interval was $120 \mathrm{mg} / \mathrm{m}^{2}$ [3]. The favorable toxicity profiles of PEP02 made it a better agent to combine with other cytotoxic agents. 5-FU/LV in combination with irinotecan was the first line treatment of colorectal cancer, which explains our interest in the evaluation of PEP02 in combination with 5 -FU/LV. The dose of weekly 5 -FU in this study was fixed as $2000 \mathrm{mg} / \mathrm{m}^{2}$, which mimicked the AIO regimen commonly used in Europe and Asia
$[10,11]$. The percentage of grade III or above AEs or all treatment-related AEs in the MTD group was lower than that in the overall safety population. For hematologic laboratory parameters, nadir was observed between days 13 and 16 after PEP02 administration; however blood biochemistry was mostly unaffected. These tolerable and manageable hematological and nonhematological toxicities indicated that this combination therapy is feasible for further application.

PEP02 affected the PK characteristics of irinotecan. Compared to the data of $125 \mathrm{mg} / \mathrm{m}^{2}$ free-form irinotecan, $80 \mathrm{mg} / \mathrm{m}^{2}$ of PEP02 showed lower Cmax (8.0 \pm $4.4 \mathrm{ng} / \mathrm{mL}$ vs. $26.3 \pm 11.9 \mathrm{ng} / \mathrm{mL})$, longer terminal $\mathrm{t} 1 / 2$ $(57.5 \pm 17.8 \mathrm{~h}$ vs. $10.4 \pm 3.1 \mathrm{~h})$ and higher AUC (343 \pm $133 \mathrm{ng} / \mathrm{mL}^{*}$ hr vs. $229 \pm 108 \mathrm{ng} / \mathrm{mL}^{*} \mathrm{hr}$ ) of SN-38 [12, 13]. These favorable PK parameters indicated that PEP02 
could decrease the influx of SN-38 from the central compartment to the peripheral, leading to less treatmentrelated toxicities, even in combination with 5-FU/LV. The PK data showed the dose-dependent linear distribution of CPT-11 when study doses were increased from 60 to $120 \mathrm{mg} / \mathrm{m}^{2}$, but no statistically significant difference was observed in the mean values of pharmacokinetic parameters of CPT-11 and SN-38, including dose-normalized $\mathrm{C}_{\text {max }}$, AUC parameters, $\mathrm{t}_{1 / 2}$, CL, and $\mathrm{V}_{\mathrm{ss}}$, possibly owning to narrow dose increments, small sample size and high inter-individual variability.

The UGT1A1 gene encoded a varied spectrum of active enzymes that are responsible for drug metabolism, including UGT. The $U G T 1 A 1 * 28$ allele is characterized by the presence of a 7th dinucleotide repeat in the TATA box of the promoter region, compared to the UGT1A1*1 allele with 6 repeats. This increased number of repeats results in the reduction in the expression of UGT, leading to decreased SN-38 detoxification and prolonged exposure time of $\mathrm{SN}-38$ in the intestines. Thus, patients with homozygous or heterozygous UGT1A1*28 and treated with irinotecan commonly developed dose limiting neutropenia and late diarrhea [14]. Similar to UGT1A1*28 polymorphism, the UGT1A1*6 allele also can decrease the activity of the enzyme in the heterozygous or homozygous genotype. It has been reported that patients with both UGT1A1*28 and UGT1A1*6 heterozygosity were at high risk to develop irinotecan-related toxicities $[15,16]$. In our study, owning to the small sample size, a clear correlation cannot be obtained between polymorphism of UGT1A family genes and pharmacokinetic parameters or toxicity of PEP02. However, one subject with heterozygous mutation in both UGT1A1*6 and UGT1A1*28 had the highest dosenormalized AUC of SN-38 and experienced grade IV neutropenia and grade III diarrhea. To draw any firm conclusions, a PK/PD study according to polymorphism of UGT1A family genes should be performed [17].

With the limitation of being a very small sample size study of 15 efficacy evaluable population, two subjects had confirmed PR and nine subjects had SD as their best-ever responses during this study period. The tumor response rate and disease control rate were 13 and $73 \%$, respectively. In a Phase I trial, clinical efficacy cannot be defined accurately because of heterogeneous tumor types and different dose levels. Of the evaluable patients, PR was noted in a heavily treated breast cancer patient and a gastric cancer patient, and four out of five patients with pancreatic cancer had SD, implying that this combination regimen is worthy of further investigation. Indeed, PEP02 either alone or in combination with 5-FU/LV was investigated in a phase II PEP0208 study [18] and a phase III NAPOLI-1 study [19] in metastatic pancreatic cancer patients who progressed after gemcitabine-containing regimen. The
NAPOLI-1 study formed the basis for the regulatory approvals of PEP02 (Irinotecan liposome injection) by the Taiwan FDA and US FDA in October 2015.

\section{Conclusions}

This is the first trial to apply PEP02 in combination with 5-FU and LV in patients with solid tumors, and major treatment-related DLTs were myelosuppression and diarrhea. PEP02 had a lower $C_{\max }$, longer $t_{1 / 2}$ and increased $\mathrm{AUC}_{0 \rightarrow \mathrm{t}}$ of SN-38 compared to irinotecan; similar results were observed in another study on PEP02 infusion alone. The dose of $80 \mathrm{mg} / \mathrm{m}^{2}$ of PEP02 in combination with D1 and D8 infusion of 5-FU/LV with every-3-week schedule is recommended for future studies.

\section{Additional file}

Additional file 1: Table S1. Tumor type, dose level, DLT, best response and single nucleotide polymorphisms of UGT1A1 28 and UGT1A1 ${ }^{*} 6$. (DOCX 18 kb)

\section{Abbreviations}

5-FU: 5-fluorouracil; AEs: Adverse events; ANC: Absolute neutrophil count; $\mathrm{AUC}_{0 \rightarrow \infty}: \mathrm{AUC}$ through infinite time; $\mathrm{AUC}_{0 \rightarrow \mathrm{t}}$ : Plasma concentration-time curve from zero to time t; $C$ : Clearance; $C_{\text {max }}$ : Peak plasma concentration; CPT-11: Irinotecan hydrochloride; CT: Computed tomography; DLT: Doselimiting toxicity; ECOG: Eastern Cooperative Oncology Group; FDA: The Food and Drug Administration; LV: Leucovorin; MTD: Maximum tolerated dose; PG: Pharmacogenetics; PK: Pharmacokinetics; PR: Partial response;

PS: Performance score; SD: Stable disease; SN-38G: SN-38 glucuronide; STD: Standard deviation; $t_{1 / 2}$ : Elimination half-life; $T_{\max }$ : Time at which $C_{\max }$ occurred; UGT1A: UDP-glucuronosyl transferase $1 \mathrm{~A}$ isoforms; $V_{\text {ss: }}$ : Volume of distribution at steady state

\section{Acknowledgments}

We thank the patients and their families who participated in this phase I study, and also thank the medical and nursing staff of the investigational sites for the care and support of the patients in this study.

Funding

This study was supported by PharmaEngine, Inc., Taipei, Taiwan.

\section{Availability of data and materials}

The study is an industry-sponsored study. The sponsor, PharmaEngine Inc, Taipei, Taiwan, prefers to keep the raw dataset in-house. However, all the information supporting the conclusions of this article is included within the text and tables of the article and summarized in Additional file 1: Table S1.

\section{Authors' contributions}

NJC and LTC wrote the manuscript. TYC, RKH, CHW and LTC enrolled the patients. NJC, YWW, CGY and LTC collected and analyzed data. YWW, CGY and LTC conceived of the study, participated in its design and coordination. All authors contributed to and approved the final version of the manuscript.

\section{Competing interests}

NJC, TYC, RKH, JYC, and CHW report no conflicts of interests. YWW and CGY are full-time employees of PharmaEngine. LTC has received an honorarium from PharmaEngine for an advisory board.

\section{Consent for publication}

Not applicable.

\section{Ethics approval and consent to participate}

The protocol and all recruiting materials and consent foam had been approved by the Joint Institutional Review Board (JIRB), covering all the participating hospitals in the study including Tri-Service General Hospital, 
Mackay Memorial Hospital, Linkou Chang Gung Memorial Hospital, National Cheng Kung University Hospital, and Kaohsiung Medical University This study had been performed in accordance with International Conference on Harmonization Good Clinical Practice guidelines, Good Clinical Laboratory Practice, and the Declaration of Helsinki. All participants from each institutions provided written informed consent.

\section{Author details}

${ }^{1}$ National Institute of Cancer Research, National Health Research Institutes, 2F, No. 367, Sheng-Li Road, Tainan 704, Taiwan. ²Division of Hematology/ Oncology, Department of Internal Medicine, National Cheng Kung University Hospital, Tainan, Taiwan. ${ }^{3}$ Division of Hematology and Oncology, Taipei Medical University-Shuang Ho Hospital, Taipei, Taiwan. ${ }^{4}$ Division of Hematology and Oncology, Department of Internal Medicine, Mackay Memorial Hospital, Taipei, Taiwan. ${ }^{5}$ Division of Hematology/Oncology, Department of Internal Medicine, Chang Gung Memorial Hospital, Linkou Taiwan. ${ }^{6}$ PharmaEngine, Inc, Taipei, Taiwan. ${ }^{7}$ Department of Internal Medicine, Kaohsiung Medical University Hospital, Kaohsiung Medical University, Kaohsiung, Taiwan.

Received: 2 March 2016 Accepted: 28 October 2016

Published online: 21 November 2016

\section{References}

1. Drummond DC, Noble CO, Guo Z, Hong K, Park JW, Kirpotin DB. Development of a highly active nanoliposomal irinotecan using a novel intraliposomal stabilization strategy. Cancer Res. 2006;66(6):3271-7.

2. Tsai CS, Park JW, Chen LT. Nanovector-based therapies in advanced pancreatic cancer. J Gastrointest Oncol. 2011;2(3):185-94.

3. Chang TC, Shiah HS, Yang $\mathrm{CH}_{\text {, }}$ Yeh KH, Cheng AL, Shen BN, Wang YW, Yeh CG, Chiang NJ, Chang JY et al. Phase I study of nanoliposomal irinotecan (PEP02) in advanced solid tumor patients. Cancer Chemother Pharmacol. 2015;75(3):579-86.

4. Saltz LB, Cox JV, Blanke C, Rosen LS, Fehrenbacher L, Moore MJ, Maroun JA, Ackland SP, Locker PK, Pirotta N et al. Irinotecan plus fluorouracil and leucovorin for metastatic colorectal cancer. Irinotecan Study Group. N Engl J Med. 2000:343(13):905-14.

5. Douillard JY, Cunningham D, Roth $A D$, Navarro M, James RD, Karasek $P$, Jandik P, Iveson T, Carmichael J, Alakl M et al. Irinotecan combined with fluorouracil compared with fluorouracil alone as first-line treatment for metastatic colorectal cancer: a multicentre randomised trial. Lancet. 2000; 355(9209):1041-7.

6. Kambe M, Kikuchi H, Gamo M, Yoshioka T, Ohashi Y, Kanamaru R. Phase I study of irinotecan by 24-h intravenous infusion in combination with 5fluorouracil in metastatic colorectal cancer. Int J Clin Oncol. 2012;17(2):150-4.

7. Iyer L, King CD, Whitington PF, Green MD, Roy SK, Tephly TR, Coffman BL, Ratain MJ. Genetic predisposition to the metabolism of irinotecan (CPT-11). Role of uridine diphosphate glucuronosyltransferase isoform 1A1 in the glucuronidation of its active metabolite ( $\mathrm{SN}-38)$ in human liver microsomes. J Clin Invest. 1998;101(4):847-54.

8. Hoskins JM, McLeod HL. UGT1A and irinotecan toxicity: keeping it in the family. J Clin Oncol. 2009;27(15):2419-21. doi: 24101200/JCO200824209478 Epub.

9. Fuchs CS, Moore MR, Harker G, Villa L, Rinaldi D, Hecht JR. Phase III comparison of two irinotecan dosing regimens in second-line therapy of metastatic colorectal cancer. J Clin Oncol. 2003;21(5):807-14.

10. Kohne CH, Wils J, Lorenz M, Schoffski P, Voigtmann R, Bokemeyer C, Lutz M, Kleeberg C, Ridwelski K, Souchon R et al. Randomized phase III study of high-dose fluorouracil given as a weekly 24-h infusion with or without leucovorin versus bolus fluorouracil plus leucovorin in advanced colorectal cancer: European organization of Research and Treatment of Cancer Gastrointestinal Group Study 40952. J Clin Oncol. 2003;21(20):3721-8.

11. Chen LT, Liu TW, Wu CW, Chung TR, Shiah HS, Jan CM, Liu JM, Whang-Peng J, Chang JY. A phase I study of weekly docetaxel, 24-h infusion of high-dose fluorouracil/leucovorin and cisplatin in patients with advanced gastric cancer. Oncology. 2002;63(3):239-47.

12. Rivory LP, Haaz MC, Canal P, Lokiec F, Armand JP, Robert J. Pharmacokinetic interrelationships of irinotecan (CPT-11) and its three major plasma metabolites in patients enrolled in phase 1/II trials. Clin Cancer Res. 1997;3(8):1261-6.
13. Rothenberg ML, Kuhn JG, Burris 3rd HA, Nelson J, Eckardt JR, Tristan-Morales M, Hilsenbeck SG, Weiss GR, Smith LS, Rodriguez Gl et al. Phase I and pharmacokinetic trial of weekly CPT-11. J Clin Oncol. 1993;11(11):2194-204.

14. Palomaki GE, Bradley LA, Douglas MP, Kolor K, Dotson WD. Can UGT1A1 genotyping reduce morbidity and mortality in patients with metastatic colorectal cancer treated with irinotecan? An evidence-based review. Genet Med. 2009;11(1):21-34. doi:10.1097/GIM.1090b1013e31818efd31877.

15. Minami H, Sai K, Saeki M, Saito Y, Ozawa S, Suzuki K, Kaniwa N, Sawada J, Hamaguchi T, Yamamoto $\mathrm{N}$ et al. Irinotecan pharmacokinetics/ pharmacodynamics and UGT1A genetic polymorphisms in Japanese: roles of UGT1A1 6 and 28. Pharmacogenet Genomics. 2007:17(7):497-504.

16. Shimoyama S. Pharmacogenetics of irinotecan: An ethnicity-based prediction of irinotecan adverse events. World J Gastrointest Surg. 2010;2(1): 14-21. doi:10.4240/wjgs.v4242.i4241.4214.

17. Saif MW. MM-398 achieves primary endpoint of overall survival in phase III study in patients with gemcitabine refractory metastatic pancreatic cancer. JOP. 2014;15(3):278-9. doi: 210.6092/1590-8577/2507.

18. Ko AH, Tempero MA, Shan Y, Su W, Lin Y, Dito E, Ong A, Yeh CG, Chen L. A multinational phase II study of liposome irinotecan (PEP02) for patients with gemcitabine-refractory metastatic pancreatic cancer. Br J Cancer. 2013;109: 920-5.

19. Wang-Gillam A, Li CP, Bodoky G, Dean A, Shan YS, Jameson G, Macarulla T, Lee KH, Cunningham D, Blanc JF, et al. NAPOLI-1 Study Group. Nanoliposomal irinotecan with fluorouracil and folinic acid in metastatic pancreatic cancer after previous gemcitabine-based therapy (NAPOLI-1): a global, randomised, open-label, phase 3 trial. Lancet. 2016;387(10018):545-57.

\section{Submit your next manuscript to BioMed Central and we will help you at every step:}

- We accept pre-submission inquiries

- Our selector tool helps you to find the most relevant journal

- We provide round the clock customer support

- Convenient online submission

- Thorough peer review

- Inclusion in PubMed and all major indexing services

- Maximum visibility for your research

Submit your manuscript at www.biomedcentral.com/submit

CioMed Central 